\title{
Hardening by softening in a flow of chainlike self-driven objects
}

Takashi Mashiko*, Yasuhito Imanishi, Ryota Kuwajima, Takashi Nagatani

Department of Mechanical Engineering, Shizuoka University, Hamamatsu 432-8561, Japan

\begin{abstract}
A two-dimensional square lattice system, on which flexible, chainlike, selfdriven objects move randomly but are drifted to a same direction, causing a unidirectional net flow, is investigated by numerical simulations. It is shown that the objects exhibit a freezing transition from a smoothly flowing state to a completely jammed state, in which the objects get immobile and cannot move anymore. Comparison with the flow of rigid objects shows that this complete jamming (hardening) results from the flexibility (softening) of each self-driven object. This is the first report of the freezing transition of a free transport system (without obstacles) where the net flow is not multidirectional (as in the case of opposing flows or crossing flows) but unidirectional.
\end{abstract}

Key words: jamming transition, traffic flow, lattice-gas model PACS: 45.70.Vn, 89.40.-a, 05.90.+m

\section{Introduction}

Pedestrian and vehicular traffic flows have attracted much interest of physicists in recent years [1-5]. Many works have been done, motivated by familiar but tough questions: Why do "phantom traffic jams" occur on expressways without traffic lights or obstacles? Are there any effective ways to maximize the current of a traffic flow under a given condition? What differences in the (macroscopic) flow dynamics do the (microscopic) properties of individual vehicles or pedestrians make? Various phenomena have been

\footnotetext{
*Corresponding author

Email address: ttmasik@ipc.shizuoka.ac.jp (Takashi Mashiko)
} 
investigated to date; pedestrian counter flows in a channel [6-15], crossing flows [16-19], evacuation processes [7, 20-28], turbulent flows of crowd [29], and flows of multiple types of mobile objects (e.g., rodlike objects of different lengths [30, 31] and a large object among small pointlike objects [32, 33]). Also, effects on traffic flows of various factors, such as social force [34, 35], fluctuations [36], bottlenecks [37, 38], traffic lights [39-41], "pheromone" [4244], and vehicular restrictions [45], have been studied. These phenomena have been modeled by various methods like cellular automata, lattice-gas methods, molecular dynamics, fluid-dynamic approaches, and mean-field models. The wide variety of the phenomena and of the methods indicates the importance and appeal of traffic flows.

In previous models mobile objects have been mimicked by pointlike objects in most cases, or at most by rigid bodies, if not pointlike (i.e., rodlike or bulky). Traffic flows of mobile objects that are deformable have little been investigated so far. In real life, however, there are various deformable mobile objects around us. Even for one-dimensional (chainlike) objects alone, examples can be given from those living, such as some kinds of bacteria and worms, to those nonliving, such as container dollies towed by a tractor and snakelike robots. Especially, studying the behaviors of deformable machines is of increasing importance, in view of the rapid factory automation and the recent development of snakelike robots [46]. Therefore, it is a challenging issue to examine the behavior of deformable mobile objects. In this paper, we give a simple lattice-gas model of unidirectional net flow of flexible, chainlike, self-driven objects, which is not just an extension of but should be distinguished from the previous models, in that it introduces the deformability or flexibility of self-driven objects. Based on the model, we investigate the dynamics of the traffic flow of flexible mobile objects by numerical simulations on a square lattice.

\section{Model and simulation}

The model considered in this article originates from the "flexible chainlike walker" (FCW) model which has been proposed recently by one of the authors (T. M.) [47]. A mobile object of length $l$ consists of $l$ serially concatenated particles, which, on a square lattice, occupy $l$ horizontally or vertically adjacent sites. One of the edge particles (the first particle) represents the head of the object and the other (the $l$ th particle) represents the tail. At $t=0, N$ objects of length $l$ are placed at random positions in a channel of 
$L$ sites in length and $W$ sites in width, each of which is put straight along the channel facing in the $+x$ direction (i.e., with its head particle rightmost). Then all the $N$ objects are updated in random order at every time step. The basic idea of updating is as follows (see Fig. 1, which schematically exemplifies the movement of an $l=5$ object): At each time step the head particle chooses one of its four nearest neighbor sites stochastically and moves to that site in a manner specifically described below. Then the subsequent particles follow the head particle. That is, the second particle moves to the site that the head particle has just left, the third particle moves to the site that the second particle has just left, and so forth.

For simplicity, the model of "naive" objects should be mentioned before the description of the model of "smart" objects which is mainly studied in the present work. To demonstrate the effect of the flexibility of objects, we also conduct simulations of "rigid" objects. In the following subsections, we describe the specific models of naive, smart, and rigid objects respectively.

\subsection{Naive objects}

The model of naive objects is simply a biased version of the original FCW model. That is, at each time step, the head particle of an object chooses one of the four nearest neighbor sites with probabilities given by

$$
p_{+x}=D+\frac{1-D}{4}, \quad p_{-x}=p_{+y}=p_{-y}=\frac{1-D}{4},
$$

where $p_{ \pm x}$ and $p_{ \pm y}$ respectively denote the probabilities of choosing the nearest neighbor site in the $\pm x$ and $\pm y$ directions, and $D$, the drift coefficient, indicates the strength of the bias into the preferred $(+x)$ direction. Note that $D=0$ corresponds to the original FCW model $\left(p_{ \pm x}=p_{ \pm y}=1 / 4\right)$ and $D=1$ corresponds to the case in which each object always chooses the nearest neighbor site on the right-hand side. If the site chosen by the head particle is occupied by another particle (either of the same object or of another), the object does not move at that time step. It should be mentioned that at least one nearest neighbor site is always occupied by the second particle (next to the head particle) of the same object, except for $l=1$.

\subsection{Smart objects}

In the case of the smart objects, in contrast to the naive objects, each object always moves unless impossible. That is, the head particle of an object chooses one of its vacant nearest-neighbor sites, except when all the four are 
occupied (in which case the object does not move at that time step). The probabilities of choosing the nearest neighbor sites, $p_{ \pm x}^{\prime}$ and $p_{ \pm y}^{\prime}$, are given by

$$
p_{i}^{\prime}= \begin{cases}p_{i}\left(1+\frac{\sum_{l \text { (occupied) }} p_{l}}{\sum_{k \text { (vacant) }} p_{k}}\right) & \text { (for vacant site) } \\ 0 & \text { (for occupied site) }\end{cases}
$$

where the summation for $k$ and $l$ is taken for vacant and occupied nearest neighbor sites, respectively. For example, when the nearest neighbor site in the $+x$ direction is occupied and the other three are vacant, the probabilities become

$$
p_{+x}^{\prime}=0, \quad p_{-x}^{\prime}=p_{+y}^{\prime}=p_{-y}^{\prime}=\frac{1}{3} .
$$

Also, when the nearest neighbor sites in $\pm y$ directions are occupied and those in $\pm x$ directions are vacant, we have

$$
\begin{aligned}
& p_{+x}^{\prime}=\left(D+\frac{1-D}{4}\right) \frac{2}{1+D}, \\
& p_{-x}^{\prime}=\frac{1-D}{4} \cdot \frac{2}{1+D}, \\
& p_{+y}^{\prime}=p_{-y}^{\prime}=0 .
\end{aligned}
$$

Equation (2) may appear to be rather complicated, but the idea is quite simple: It is just designed so that the probability distribution of $p_{i}^{\prime}$ for vacant sites should be proportional to that of $p_{i}$ in Eq. (1), with an additional condition of $\sum_{k \text { (vacant) }} p_{i}^{\prime}=1$. In other words, both the head particles of a naive object and of a smart object try to choose a nearest neighbor site in accordance with Eq. (1), where the only difference is that the former tries the choice one time only, while the latter tries repeatedly until it chooses a vacant site (unless impossible), at each time step.

\subsection{Rigid objects}

The model of the rigid objects is as follows. At each time step, a rigid object of length $l$ moves one site in either of the four directions with its shape 
fixed. The probabilities of moving in the $\pm x$ and $\pm y$ directions, $p_{ \pm x}^{\prime \prime}$ and $p_{ \pm y}^{\prime \prime}$, are given by the following formula of the same form as Eq (2);

$$
p_{i}^{\prime \prime}= \begin{cases}p_{i}\left(1+\frac{\sum_{l(\text { closed })} p_{l}}{\sum_{k \text { (open })} p_{k}}\right) & \text { (for open direction) } \\ 0 & \text { (for closed direction) }\end{cases}
$$

where the $\pm y$ direction is regarded as open if and only if all the $l$ neighboring sites on the $\pm y$ side are vacant. If any of the $l$ neighboring sites is occupied, the object does not move into that closed direction at that time step. In the sense that they move whenever there are any open directions, the rigid objects are rather "smart" than "naive".

\subsection{Parameters}

To describe the behaviors of the objects, we define the following quantities: The density of particles is the ratio of the total number of particles to the number of sites in the channel;

$$
\rho=\frac{l N}{L W}
$$

The mean velocity is defined as

$$
v(t)=\frac{n_{+}(t)-n_{-}(t)}{l N},
$$

where $n_{ \pm}(t)$ is the number of particles that have moved in the $\pm x$ direction at time $t$ (note that $\left.n_{+}(t)+n_{-}(t) \leq l N\right)$. The net current in the $+x$ direction is given by

$$
Q(t)=\rho v(t)=\frac{n_{+}(t)-n_{-}(t)}{L W} .
$$

It should be noted that $v(t)$ and $Q(t)$ are defined here in terms of the number of all the particles that have moved, not the number of the head particles. The two definitions coincide in case of the rigid objects, where $n_{ \pm}(t)$ is exactly $l$ times the number of the head particles that have moved in the $\pm x$ direction. For the flexible objects, however, the two definitions generally give different values of $v(t)$ and $Q(t)$, although they may be expected to coincide when averaged over a sufficient time in a steady state. 


\section{Results and discussion}

We conducted simulations under the above-described rules and under periodic boundary conditions (for both the horizontal and vertical boundaries). It was found that in general the velocity $v$ and current $Q$ decrease with time, to asymptotic values $v_{\infty}=v(t \rightarrow \infty)$ and $Q_{\infty}=Q(t \rightarrow \infty)$, respectively. Typically, $t \sim 10000$ was enough for $v$ and $Q$ to reach the asymptotic values. Now we will show the results of simulations. The data shown in this article are for $L=100, W=20$, and $D=0.9$, unless otherwise noted.

In Fig. 2, the values of $v_{\infty}$ and $Q_{\infty}$ are plotted as functions of $\rho$ for $1 \leq l \leq 8$ smart objects, each of which was averaged over 20000 time steps in the asymptotic state $(t=30001-50000)$ and over at least 100 runs (consequently, the number of samples is at least $2 \times 10^{6}$ ). For $l=1, v_{\infty}$ remains roughly constant, while $Q_{\infty}$ increases linearly, with increasing $\rho$ in the lower- $\rho$ region $(0<\rho \lesssim 0.3)$, then $v_{\infty}$ begins to decrease gradually, while $Q_{\infty}$ peaks at around $\rho \sim 0.45$, and finally both decrease with increasing $\rho$. Similar results are obtained for $l=2$. On the other hand, for $l \geq 3$, the behaviors are qualitatively different from those for $l \leq 2$ : Although $v_{\infty}$ stays nearly constant and $Q_{\infty}$ increases linearly in the lower- $\rho$ region as in the case of $l \leq 2$, they drop sharply and settle in a jammed state. This state can be referred to as a "complete" jamming, in the sense that $v_{\infty}$ and $Q_{\infty}$ become naught and this lasts ever since. We also notice that the longer the object is, the smaller the critical density at which the jamming transition occurs is. Furthermore, Fig. 2 implies that the curves for $l \geq 3$ can be divided into three groups by the critical density: $l=3$ and $4 ; l=5,6$, and 7 ; and $l=8$. The uniqueness of the freezing transition to the completely jammed state observed here and the interpretations of the results will be discussed later.

To highlight the effect of the flexibility, Fig. 3 shows the plots of $v_{\infty}$ and $Q_{\infty}$ for the rigid objects. For $l=1$, the result is equivalent with that of the flexible objects shown in Fig. 2. This is because the $l=1$ objects consists of a single particle hence it makes no sense to consider the flexibility, and the rule of movements of the rigid object is essentially smart-object-like, as was noted previously. Except for $l=1$, however, the behaviors of the rigid objects are obviously different from those of the flexible objects. For $l=2$, it is apparent that both $v_{\infty}$ and $Q_{\infty}$ are larger than those of the flexible objects in the higher- $\rho$ region: The decrease of $v_{\infty}$ is more gradual and $Q_{\infty}$ continues to increase instead of reaching a peak. This tendency is more pronounced for $l \geq 3: v_{\infty}$ remains nearly constant (or rather increases a little) and $Q_{\infty}$ 
increases almost linearly in the whole $\rho$-region investigated. This is quite understandable: The longer the object is (or the larger the density is), the more likely the lateral movements (in the $\pm y$ directions) are restricted due to the higher probabilities of the existence of other objects in the neighboring sites. Thus, longer rigid objects are more likely to move forward in the $+x$ direction rather than in the other directions.

For reference, similar plots of $v_{\infty}$ and $Q_{\infty}$ for flexible but naive objects are also shown in Fig 4. These plots are qualitatively different from those of the smart objects or of the rigid objects: In comparison with the results of the smart objects (Fig. 2), we notice that the drops of the curves are neither sharp nor smooth. Probably, this difference results from the fact that while the smart object always moves whenever possible, the naive object does not move if its head particle happens to choose an occupied nearest neighbor site and hence possesses higher degree of randomness. It is interesting that the values of $v_{\infty}$ and $Q_{\infty}$ of the naive objects are larger than those of the smart objects in the higher- $\rho$ region (for $l=1,2$, and $l=3$ in particular), whereas the converse relations can be seen in the lower- and middle- $\rho$ regions.

Here, the effects of the channel length $L$, width $W$, and the drift coefficient $D$ should be briefly mentioned. The dependence of $v_{\infty}$ and $Q_{\infty}$ on $L, W$, and $D$ for smart objects are shown in Figs. 5, 6, and 7, respectively, for $l=3$ smart objects. We see that the shape of the $v_{\infty}-\rho$ or $Q_{\infty}-\rho$ curves does not change basically when $L$ is varied. The $v_{\infty^{-}}$or $Q_{\infty}$-values before and after the transition are the same for all $L$. Only the transition point depends on $L$; the larger $L$ is, the lower the critical density is. We did not observe a sign of saturation of the critical density within the present simulations up to $L=1600$. Likewise, the shape of the $v_{\infty^{-}} \rho$ or $Q_{\infty^{-}} \rho$ curves does not depend on $W$, except for the critical density. The objects undergo the transition at a lower density in the case of $W=5$, that is, when the channel width is narrow, being comparable with the length $l=3$ of the object. Except for this special case, however, the critical density is relatively large, and decreases as $W$ increases. In addition, the saturation of the critical density was observed (see the plots for $W=160$ and 320). As for the dependence on $D$, we see that the larger $D$ is, the larger the nearly constant value of $v_{\infty}$ in the lower- $\rho$ region, the peak value of $Q_{\infty}$, and the critical density are. Therefore, it can be concluded that the increase of the drift results in the increase of the net flow, which is quite intuitive. In this article, typical results for $L=100$, $W=20$, and $D=0.9$ have been presented, since the results do not change qualitatively depending on $L, W$, and $D$, while they considerably depend on 
the flexibility or the length $l$ of objects.

Now, let us discuss the results of the smart objects (Fig. 2). To clearly demonstrate the effect of the flexibility of objects, we show typical pattern variations with time of the smart and the rigid objects $(l=3)$ in Figs. 8 and 9 , respectively. Snapshots at $t=0,500,1000,2000,3000,4000,5000,10000$ (from top to bottom) for $\rho=0.30,0.45,0.60$ (from left to right) are shown. At $\rho=0.30$, the smart objects, on an average, proceed smoothly in the $+x$ direction, and the situation does not change with time. Thus in the lower- $\rho$ region represented by $\rho=0.30, v_{\infty}$ keeps a nearly constant, high value and $Q_{\infty}$ increases almost linearly with $\rho$. But at $\rho=0.60$, the flow comes to slow down with time, until the objects finally settle in the completely jammed state. Therefore, in the higher- $\rho$ region represented by $\rho=0.60, v_{\infty}$ and $Q_{\infty}$ approach $v_{\infty}=0$ and $Q_{\infty}=0$, respectively. In the middle- $\rho$ region represented by $\rho=0.45$, the objects sometimes fall into the jammed state as in the higher- $\rho$ region, and other times continue to move forward as in the lower- $\rho$ region, in the finite length of the simulation time. Hence, we observe the freezing transition (hardening) from the smoothly flowing state (characterized by higher $v_{\infty}$ and linearly increasing $Q_{\infty}$ ) to the completely jammed state (characterized by $v_{\infty}=Q_{\infty}=0$ ). On the other hand, the rigid objects do not exhibit either that kind of jamming or apparent changes with time but just move forward smoothly for all the three values of density; $\rho=0.30,0.45$, and 0.60 . Therefore, as was shown in Fig. $3, v_{\infty}$ keeps a nearly constant, high value and $Q_{\infty}$ increases linearly with $\rho$ in the whole region of $\rho$ investigated.

What causes this crucial difference? Observing the time variations of patterns in detail, we notice that the flexible objects sometimes form a scrum when they happen to encounter each other, and get locked by themselves. Such a situation was found in the many-body system of FCWs and was named "mutual locking" [47]. Figure 10 shows some examples of mutual-locking patterns: Four $l=3$ or longer flexible objects can form a fylfot scrum (a). Flexible objects of $l=5$ or longer can form coupler-shaped gridlock when two of them happen to meet (b). Furthermore, if the object is long enough $(l \geq 8)$, it can lock itself by coiling (c), which is, so to speak, "self-locking". Once mutual locking (including self-locking) takes place, the objects concerned can never move again. This nucleates the jamming transition, and is the origin of the "completeness" of the jamming. A body of mutual locking generated acts as an obstacle and dams up the flow of other objects. The objects blocked by the obstacle then becomes part of the obstacle and block other objects 
that follow. It is possible but not likely for an object blocked by an obstacle to step sideways off the obstacle and continue to move forward, since other objects are coming over one after another and obstruct such an avoiding movement. This effect is more significant when the drift is stronger and the density is higher, which probably explains the sharper drops for larger $D$ shown in Fig. 7. This way, the obstacle grows larger and larger, resulting in the formation of the completely jammed state, when $\rho$ is higher.

Now we can qualitatively explain the basic properties observed in Fig. 2. We noticed that the larger $l$ is, the lower the critical density at which the jamming transition occurs becomes. This is because more patterns of mutual locking are possible for longer objects. For example, the pattern of Fig. 10 (a) is the only one possible for $l=3$ objects (except for the "mirror-image isomer"), but for $l=4$ objects, other types of four-body mutual locking can take place in addition, as shown in Fig. 10 (d). Hence, longer objects are more likely to undergo mutual locking. Likewise, the properties of mutual locking explain the grouping of the curves in Fig. 2 which was mentioned previously: Curves for $l=3$ and 4 show a sharp drop at relatively high values of density, as only four-body mutual locking is possible, which occurs only when the density is higher. The transition points for $l=5,6$, and 7 are lower than those for $l=3$ and 4, since two-body mutual locking (Fig. 10 (b)), which is expected at lower densities, can take place in addition. Furthermore, $l=8$ objects exhibit the jamming transition at much lower density, because they can experience self-locking (Fig. 10 (c)), which can happen irrespective of the density of objects. In regard to the last point, we should expect that in the case of $l \geq 8$ the jamming transition occurs at infinitesimal density $(\rho \rightarrow 0)$ due to the occurrence of self-locking in the long-time limit $(t \rightarrow \infty)$. Even though for $D=0.9$ the transition was observed at around $\rho=0.05$ (see Fig. 2) in the present simulations of finite time length, for $D=0.3,0.5,0.7$, in which cases self-locking is more likely to occur, we checked that $v_{\infty}$ and $Q_{\infty}$ are 0 in the whole $\rho$-region investigated (data not shown).

Finally, the uniqueness of the complete jamming observed in the present study, which was possible only for flexible objects (e.g., $l \geq 3$ smart objects, see Fig. 2), should be mentioned. In previous studies of unidirectional net traffic flows, jammed states observed are "incomplete". That is, the objects (vehicles or pedestrians) continue to move slowly even in the jam, or restart to proceed freely when getting out of the jam in which they may have made a complete stop temporarily. In fact, there have been reports of complete jamming of rigid objects $[6,8-10,13-19,36]$. However, all of these completely 
jammed states are observed in systems of counter flows or crossing flows, where two or more groups of objects moving in different directions conflict. The net flow is not unidirectional but multidirectional. In the present study, on the other hand, the net flow is unidirectional, though at each time step each object can move in either of all the four directions including ones opposite or perpendicular to the drift direction. Also, it should be mentioned that in studying the traffic flow of rigid but divertible objects, we have recently found similar complete jamming [48]. The aim of that study is to clarify the effect of turning of the mobile objects flowing through obstacles, by comparing flows of divertible objects (which can turn right or left and is identical with the smart object of $l=2$ ) and of translational objects (which cannot turn but can step aside). The jamming is also of a unidirectional net flow, but observed only in the presence of obstacles; it is not observed in the absence of obstacles, as was mentioned previously in this article in comparing the behaviors of $l=2$ and $l=3$ smart objects. Therefore, we emphasize that the present result of flexible objects is the first report of a complete jamming in the simplest situation of a traffic flow; a unidirectional net flow without obstacles.

\section{Conclusion}

In summary, we presented a two-dimensional lattice-gas model of flows of flexible, chainlike, self-driven objects, and performed numerical simulations. The objects move randomly to one of the four directions but are drifted to a same direction. Objects of $l \geq 3$, which are flexible, showed a jamming transition. The jammed state observed here was a "complete" one, in the sense that the objects cannot move anymore, whereas the jammed states so far reported on unidirectional net flows were temporal or mobile (even if the block of jamming looks fixed, the objects inside it can move slowly). The present work is the first report of the freezing transition to the completely jammed state in a unidirectional net flow of objects without the presence of obstacles. Comparison of the results with those of rigid objects and the fact that the jamming transition was not observed for $l \leq 2$ revealed that the jamming transition (hardening) is attributable to the flexibility (softening) of objects. We expect that the present results exemplified the specific nature of a flow of flexible or deformable objects and motivate further investigations which may reveal other properties peculiar to flexible mobile objects. Our next simulations are on process along these lines, where we study, for exam- 
ple, counter flows, crossing flows, mixed flows of different type of objects (in respect of flexibility or length $l$ ), and flows in the presence of obstacles.

\section{References}

[1] D. E. Wolf, A. Bachem, Workshop on Traffic and Granular Flow, World Scientific, New Jersey, 1996.

[2] B. S. Kerner, The Physics of Traffic: Empirical Freeway Pattern Features, Engineering Applications, and Theory, Springer, Berlin, 2004.

[3] D. Chowdhury, L. Santen, A. Schadschneider, Phys. Rep. 329 (2000) 199.

[4] D. Helbing, Rev. Mod. Phys. 73 (2001) 1067.

[5] T. Nagatani, Rep. Prog. Phys. 65 (2002) 1331.

[6] M. Muramatsu, T. Irie, T. Nagatani, Physica A 267 (1999) 487.

[7] C. Burstedde, K. Klauck, A. Schadschneider, J. Zittartz, Physica A 295 (2001) 507.

[8] Y. Tajima, K. Takimoto, T. Nagatani, Physica A 313 (2002) 709.

[9] S. Maniccam, Physica A 321 (2003) 653.

[10] F. Weifeng, Y. Lizhong, F. Weicheng, Physica A 321 (2003) 633.

[11] M. Isobe, T. Adachi, T. Nagatani, Physica A 336 (2004) 638.

[12] R. Nagai, M. Fukamachi, T. Nagatani, Physica A 358 (2005) 516.

[13] R. Nagai, T. Nagatani, Physica A 366 (2006) 503.

[14] W. G. Weng, T. Chen, H. Y. Yuan, W. C. Fan, Phys. Rev. E 74 (2006) 036102 .

[15] M. Fukamachi, T. Nagatani, Physica A 377 (2007) 269.

[16] O. Biham, A. A. Middleton, D. Levine, Phys. Rev. A 46 (1992) R6124.

[17] M. Muramatsu, T. Nagatani, Physica A 275 (2000) 281. 
[18] M. Muramatsu, T. Nagatani, Physica A 286 (2000) 377.

[19] S. Maniccam, Physica A 363 (2006) 512.

[20] D. Helbing, I. Farkas, T. Vicsek, Nature 407 (2000) 487.

[21] H. Klüpfel, T. Meyer-König, J. Wahle, M. Schreckenberg, Theory and Practical Issues on Cellular Automata: Proceedings of the 4th International Conference on Cellular Automata for Research and Industry, 2000, p. 63.

[22] Y. Tajima, T. Nagatani, Physica A 292 (2001) 545.

[23] T. Itoh, T. Nagatani, Physica A 313 (2002) 695.

[24] A. Kirchner, A. Schadschneider, Physica A 312 (2002) 260.

[25] D. Helbing, M. Isobe, T. Nagatani, K. Takimoto, Phys. Rev. E 67 (2003) 067101.

[26] M. Isobe, D. Helbing, T. Nagatani, Phys. Rev. E 69 (2004) 066132.

[27] D. Helbing, A. Johansson, J. Mathiesen, M. H. Jensen, A. Hansen, Phys. Rev. Lett. 97 (2006) 168001.

[28] A. Varas, M. D. Cornejo, D. Mainemer, B. Toledo, J. Rogan, V. Muñoz, J. A. Valdivia, Physica A 382 (2007) 631.

[29] D. Helbing, A. Johansson, H. Z. Al-Abideen, Phys. Rev. E 75 (2007) 046109.

[30] R. Nagai, M. Fukamachi, T. Nagatani, Physica A 367 (2006) 449.

[31] S. Ito, T. Nagatani, T. Saegusa, Physica A 373 (2007) 672.

[32] R. Jiang, Q.-S. Wu, Physica A 364 (2006) 457.

[33] R. Jiang, Q.-S. Wu, Physica A 368 (2006) 239.

[34] D. Helbing, P. Molnár, Phys. Rev. E 51 (1995) 4282.

[35] W. Yu, A. Johansson, Phys. Rev. E 76 (2007) 046105.

[36] D. Helbing, I. J. Farkas, T. Vicsek, Phys. Rev. Lett. 84 (2000) 1240. 
[37] T. Nagatani, Physica A 300 (2001) 558.

[38] Y. Tajima, K. Takimoto, T. Nagatani, Physica A 294 (2001) 257.

[39] E. Brockfeld, R. Barlovic, A. Schadschneider, M. Schreckenberg, Phys. Rev. E 64 (2001) 056132.

[40] M. Sasaki, T. Nagatani, Physica A 325 (2003) 531.

[41] B. A. Toledo, V. Muñoz, J. Rogan, C. Tenreiro, Phys. Rev. E 70 (2004) 016107.

[42] D. Chowdhury, V. Guttal, K. Nishinari, A. Schadschneider, J. Phys. A 35 (2002) L573.

[43] K. Nishinari, D. Chowdhury, A. Schadschneider, Phys. Rev. E 67 (2003) 036120 .

[44] K. Nishinari, K. Sugawara, T. Kazama, A. Schadschneider, D. Chowdhury, PhysicaA 372 (2006) 132.

[45] H. K. Lee, R. Barlovic, M. Schreckenberg, D. Kim, Phys. Rev. Lett. 92 (2004) 238702.

[46] A. A. Transeth, K. Y. Pettersen, Proceedings of International Conference on Control, Automation, Robotics, and Vision, 2006, p. 1393.

[47] T. Mashiko, Phys. Rev. E 78 (2008) 011106.

[48] A. Matsui, T. Mashiko, T. Nagatani, Physica A 388 (2009) 157. 

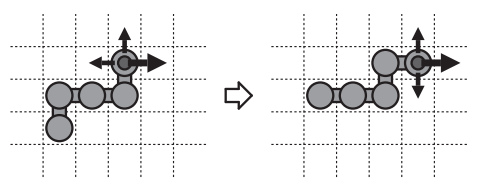

$\sqrt{3}$
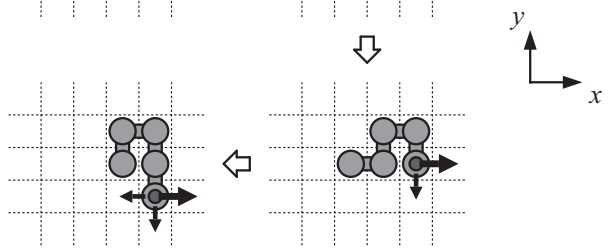

Figure 1: Model of chainlike mobile object on a square lattice. A typical series of movements of an $l=5$ object is illustrated. At each time step the head particle (represented by the double circle) moves to one of its four nearest neighbor sites, which is chosen stochastically but with some bias, followed by the subsequent particles. Possible moving directions are indicated by the arrows, one of which is the preferred $(+x)$ direction represented by the larger arrows. 

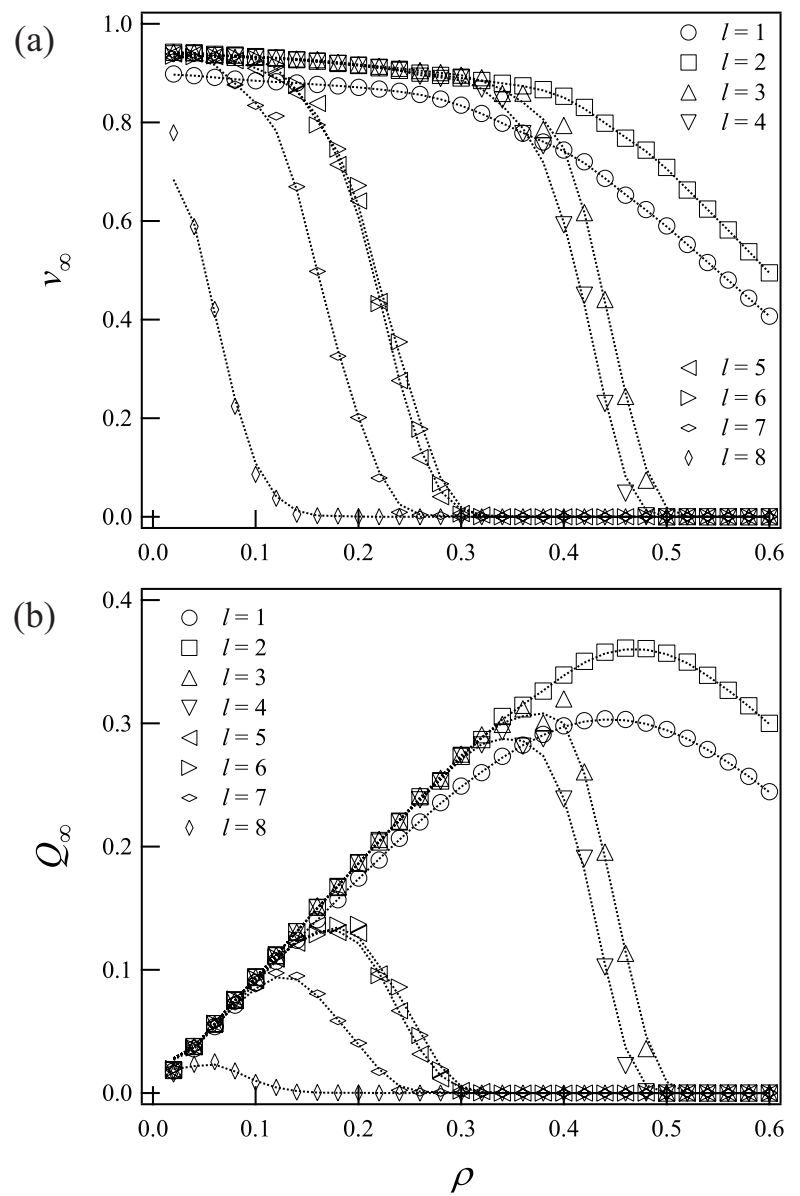

Figure 2: Asymptotic velocity $v_{\infty}$ (a) and current $Q_{\infty}$ (b) as functions of density $\rho$ for "smart" objects. Data are for $L=100, W=20, D=0.9$, and for $1 \leq l \leq 8$. Each symbol represents the averaged value taken over 20000 time steps and over at least 100 runs. Dotted lines are plotted as a guide. 
(a)

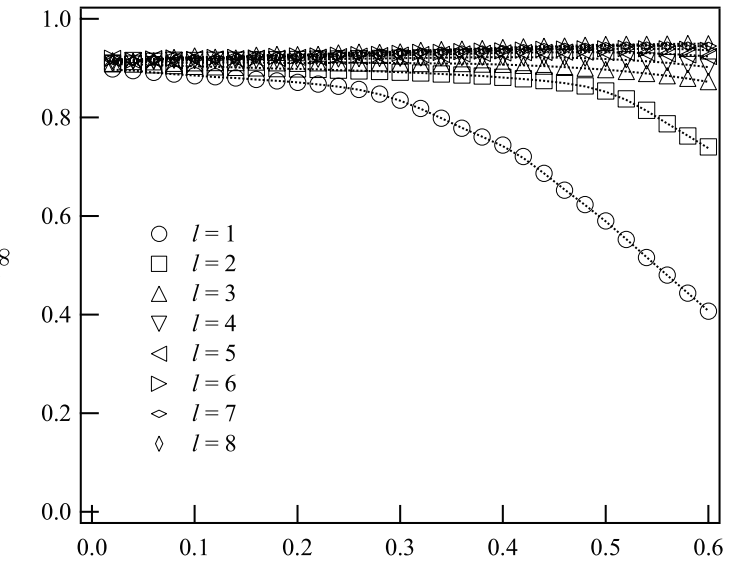

(b)

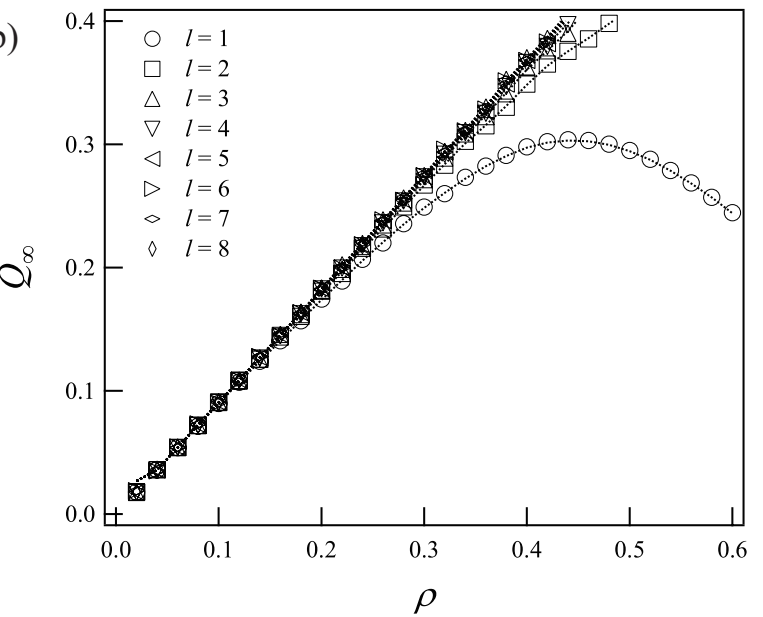

Figure 3: Asymptotic velocity $v_{\infty}$ (a) and current $Q_{\infty}$ (b) as functions of density $\rho$ for rigid objects. Data are for $L=100, W=20, D=0.9$, and for $1 \leq l \leq 8$. Each symbol represents the averaged value taken over 20000 time steps and over at least 100 runs. Dotted lines are plotted as a guide. 

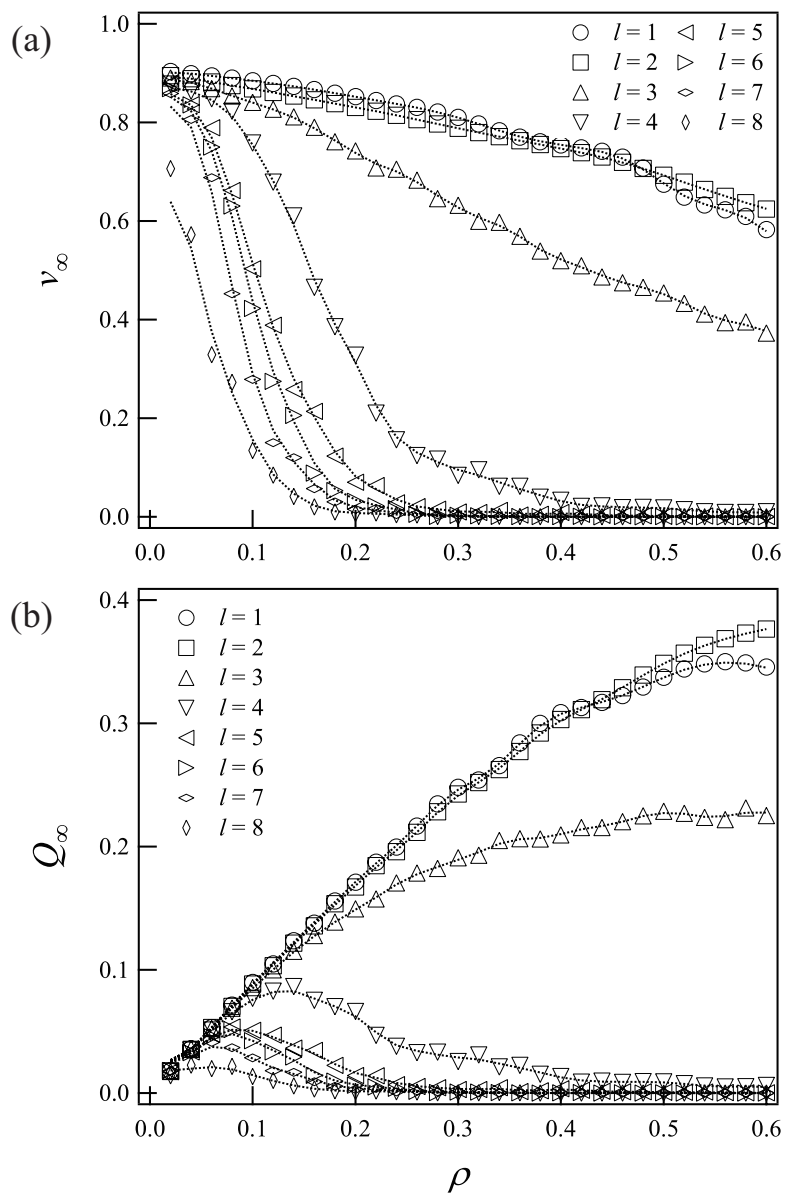

Figure 4: Asymptotic velocity $v_{\infty}$ (a) and current $Q_{\infty}$ (b) as functions of density $\rho$ for "naive" objects. Data are for $L=100, W=20, D=0.9$, and for $1 \leq l \leq 8$. Each symbol represents the averaged value taken over 20000 time steps and over at least 100 runs. Dotted lines are plotted as a guide. 

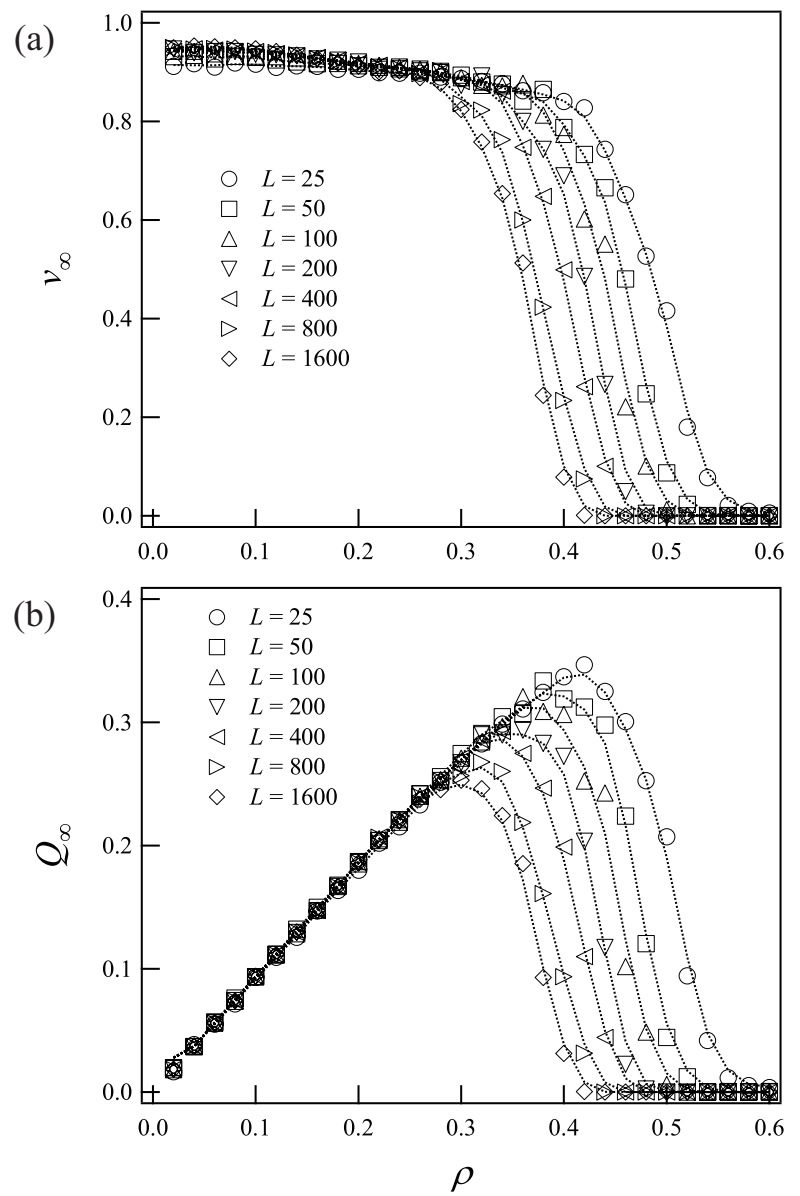

Figure 5: Asymptotic velocity $v_{\infty}$ (a) and current $Q_{\infty}$ (b) as functions of density $\rho$ for "smart" objects. Data are for $l=3, W=20, D=0.9$ and for $L=$ $25,50,100,200,400,800,1600$. Each symbol represents the averaged value taken over 20000 time steps and over at least 100 runs. Dotted lines are plotted as a guide. 


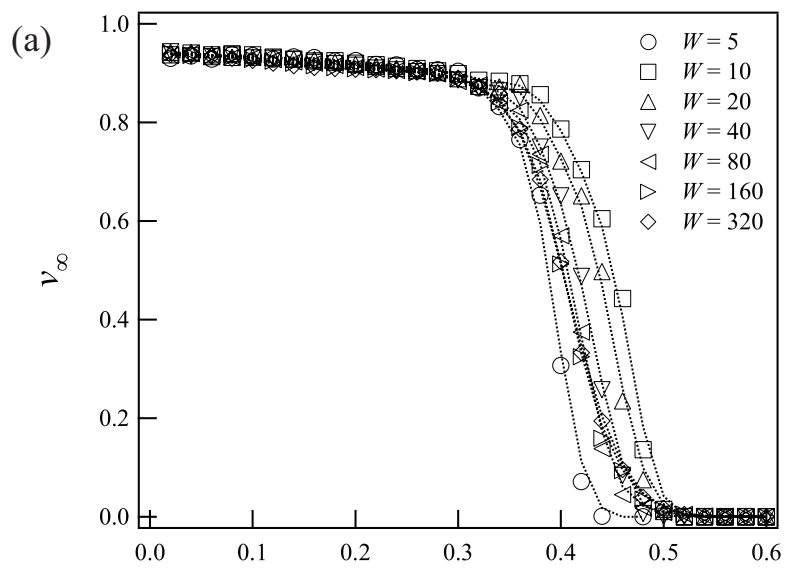

(b)

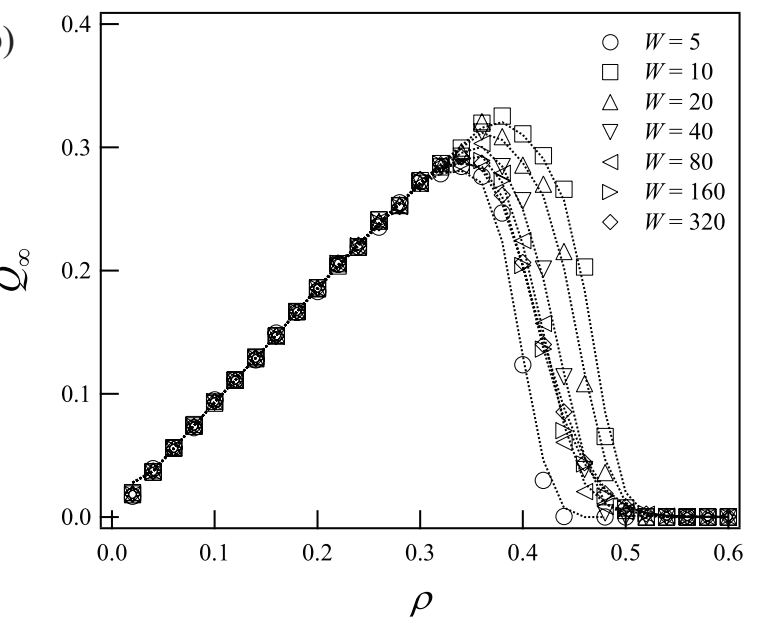

Figure 6: Asymptotic velocity $v_{\infty}$ (a) and current $Q_{\infty}$ (b) as functions of density $\rho$ for "smart" objects. Data are for $l=3, L=100, D=0.9$, and for $W=$ $5,10,20,40,80,160,320$. Each symbol represents the averaged value taken over 20000 time steps and over at least 100 runs. Dotted lines are plotted as a guide. 

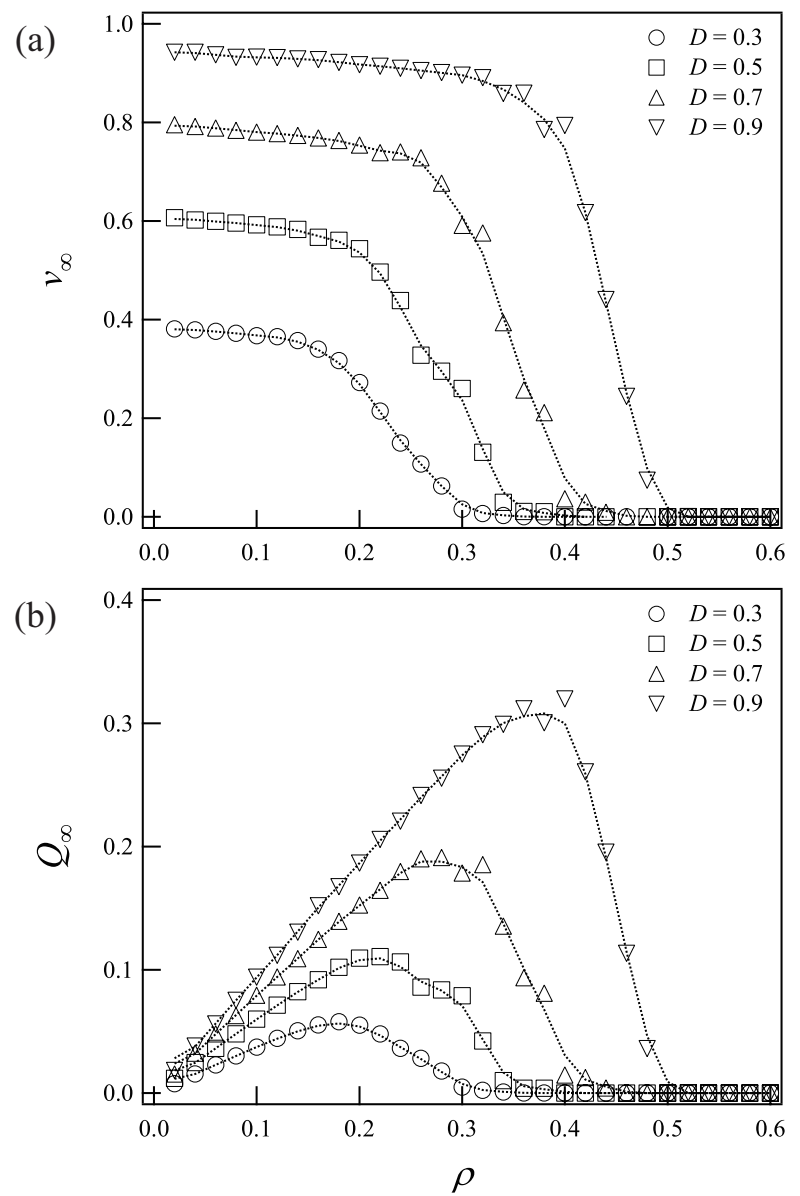

Figure 7: Asymptotic velocity $v_{\infty}$ (a) and current $Q_{\infty}$ (b) as functions of density $\rho$ for "smart" objects. Data are for $l=3, L=100, W=20$, and for $D=0.3,0.5,0.7,0.9$. Each symbol represents the averaged value taken over 20000 time steps and over at least 100 runs. Dotted lines are plotted as a guide. 

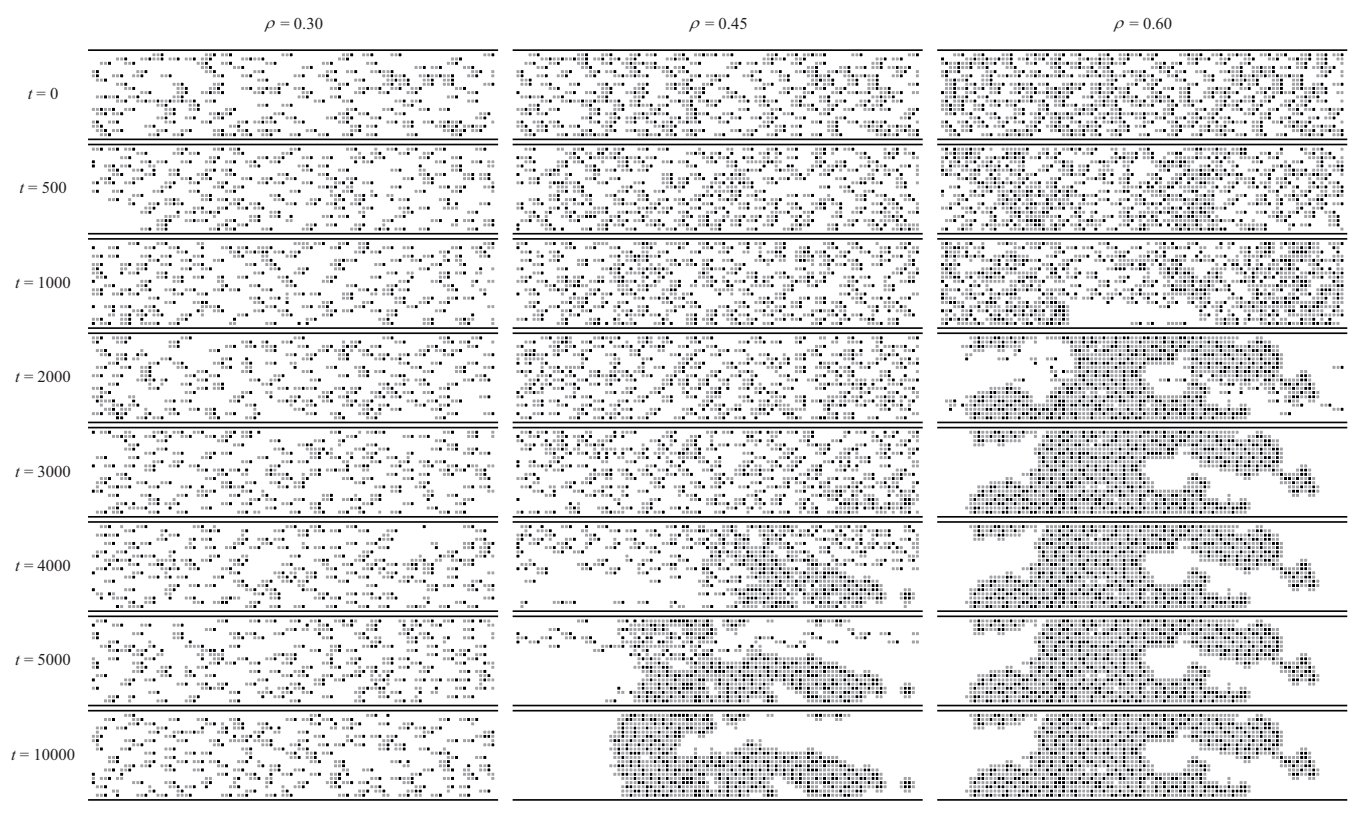

Figure 8: Typical time variations of the flow patterns of $l=3$ flexible ("smart") objects on a channel of $L=100 \times W=20$. Snapshots at $t=0,500,1000,2000,3000,4000,5000,10000$ (from top to bottom) for $\rho=0.30,0.45,0.60$ (from left to right) and $D=0.9$ are shown. The black points represent the head particles, and the gray the other particles. 

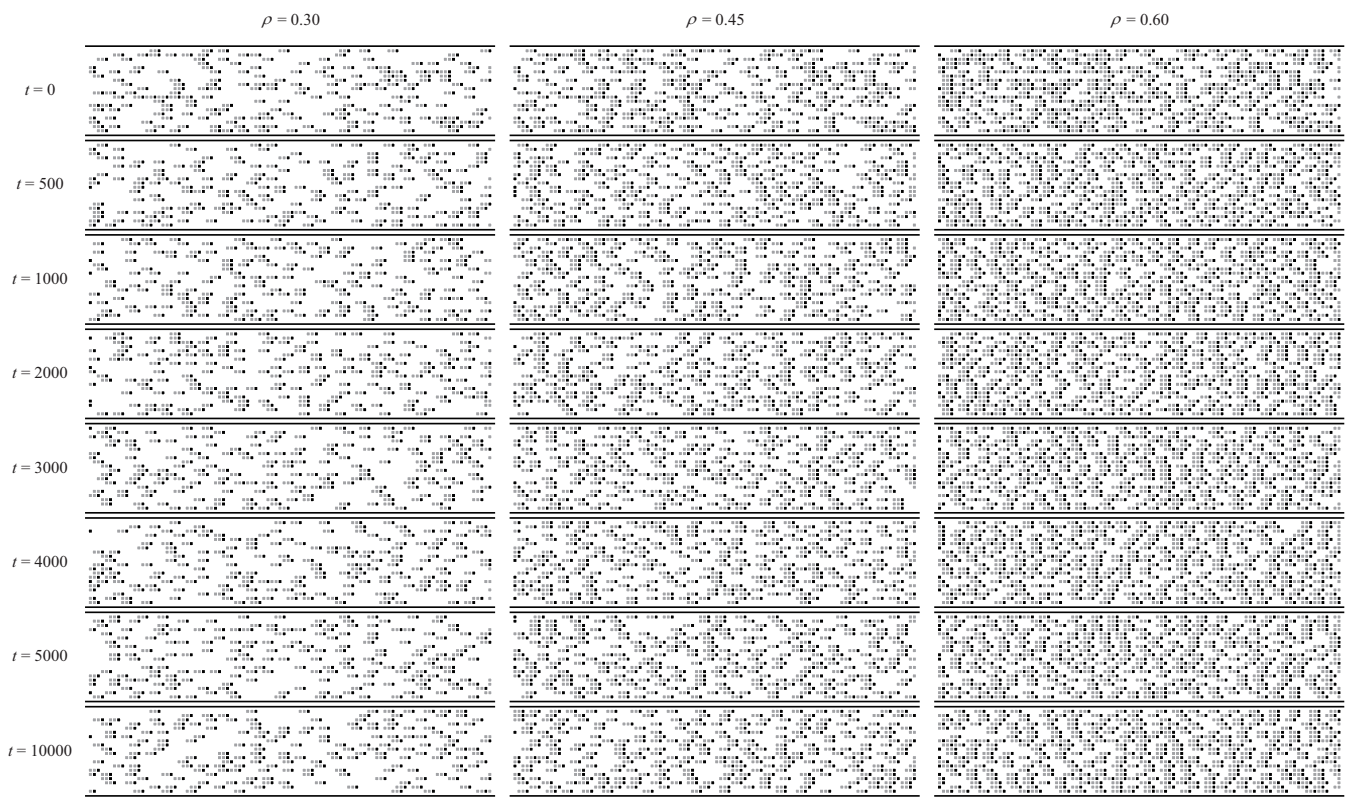

Figure 9: Typical time variations of the flow patterns of $l=3$ rigid objects on a channel of $L=100 \times W=20$. Snapshots at $t=0,500,1000,2000,3000,4000,5000,10000$ (from top to bottom) for $\rho=0.30,0.45,0.60$ (from left to right) and $D=0.9$ are shown. The black points represent the head particles, and the gray the other particles. 


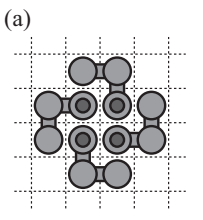

(b)

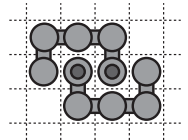

(c)

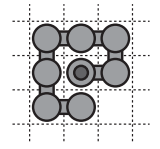

(d)

Figure 10: Typical patterns of "mutual locking" of flexible objects. (a) Four-body mutual locking of $l=3$ objects. (b) Two-body mutual locking of $l=5$ objects. (c) Self-locking of an $l=8$ object. (d) Other types of four-body mutual locking than type (a), possible for $l=4$ objects. 